\title{
Average lower independence number in splitting graphs
}

\author{
Aysun Aytac and Betul Atay \\ Department of Mathematics, Faculty of Science, Ege University
}

Received: 30 October 2016, Accepted: 9 November 2016

Published online: 17 December 2016.

\begin{abstract}
Let $G$ be a graph. The splitting graph $S^{\prime}(G)$ for a graph $G$ is obtained by adding a new vertex $v^{\prime}$ corresponding to each vertex $v$ of $G$ such that $N(v)=N\left(v^{\prime}\right)$, where $N(v)$ and $N\left(v^{\prime}\right)$ are neighborhood sets of $v$ and $v^{\prime}$, respectively. The average lower independence number $i_{a v}(G)$ of a graph $G$ is defined as $\frac{1}{|V(G)|} \sum_{v \in V} i_{v}(G)$, where $i_{v}(G)$ is the minimum cardinality of a maximal independent set that contains $v$. In this paper, we consider the average lower independence number in splitting graph. We determine the average lower independence number of $S^{\prime}(G)$ for specific graphs $G$.
\end{abstract}

Keywords: Graph vulnerability, Network design and communication, Splitting graph, Average lower independence number

\section{Introduction}

The study of the security and stability of networks plays a central role in reducing the risk and consequences of attacks or disjunctions of any type. The concept of vulnerability is very important in network analysis. The problem of measuring the vulnerability of graphs has received much attention in the field of computer or communication networks. Computer or communication networks are so designed that they do not easily get disrupted under external attack and, furthermore, are easily reconstructible if they do get disrupted. If we think of a graph as modeling a network, several vulnerability measures have been used to describe the stability of networks, including connectivity, toughness, scattering number, binding number and integrity $[2,6,8]$. Each of these parameters have been used to measure the vulnerability of networks in the case the communication vertices are damaged.

The average lower independence number of a graph is a new parameter to measure the vulnerability of networks. This parameter is closely related to the problem of finding large independent sets in graphs.

In a graph $G=(V(G), E(G))$, a subset $S \subseteq V(G)$ of vertices is a dominating set if every vertex in $V(G)-S$ is adjacent to at least one vertex of $S$. The dominating number $\gamma(G)$ is the minimum cardinality of a dominating set. The independent domination number (also called the lower independence number) $i(G)$ of $G$ is the minimum cardinality of a set that is both independent and dominating.

Henning introduced the concept of average independence. For a vertex $v$ of a graph $G$, the lower independence number, denoted by $i_{v}(G)$, is the minimum cardinality of a maximal independent set of $G$ that contains $v$. The average lower independence number of $G$, denoted by $i_{a v}(G)$, is the value $\frac{1}{|V(G)|} \sum_{v \in V} i_{v}(G)[2,3,8]$. It is clear that $i(G)=\min \left\{i_{v}(G): v \in V(G)\right\}$ and so $i(G) \leq i_{a v}(G)$.

In this paper, we consider finite undirected graphs without loops and multiple edges. Let $G=(V(G), E(G))$ be a graph 
with vertex set $V(G)$ and edge set $E(G)$. The order of $G$, denoted by $|V(G)|$, is the number of vertices in $G$. The open neighborhood of $v$ is $N(v)=\{u \in V(G): u v \in E(G)\}$ and the closed neighborhood of $v$ is $N[v]=\{v\} \cup N(v)$. For a set $S \subseteq V, N(S)=\bigcup_{v \in S} N(v)$ and $N[S]=N(S) \cup S$. An independent set of vertices of a graph $G$ is a set of vertices of $G$ whose elements are pairwise nonadjacent. The independence number $\beta(G)$ of $G$ is the maximum cardinality among all independent sets of vertices of $G[6]$.

We use $\lfloor x\rfloor$ to denote the largest integer not greater than $x$, and $\lceil x\rceil$ to denote the least integer not less than $x$. We let $x \equiv_{l} y$ mean $x \equiv y(\bmod l)$.

The paper is organized as follows. In section 2, some of the existing literature on average independent domination number is reviewed. In section 3, the average lower independence number of the splitting graph $S^{\prime}(G)$ when $G$ is a specified family of graphs is computed. Finally, section 4 concludes the paper.

\section{Basic results}

In this section, we will review some of the known result on average lower independence number.

Theorem 1.[5,8] For every vertex $v$ in a graph,
(a) $i(G) \leq i_{v}(G) \leq \beta(G)$
(b) $i(G) \leq i_{a v}(G) \leq \beta(G)$.

Theorem 2.[5] For any graph $G$ of order $n$ with independent domination number $i$ and independence number $\beta$,

$$
i_{a v}(G) \leq \beta-\frac{i(\beta-i)}{n}
$$

Theorem 3.[8] If $T$ is a tree of order $n \geq 2$, then

$$
i_{a v}(G) \leq n-2+\frac{2}{n}
$$

Theorem 4.[2] Let $G_{1}$ and $G_{2}$ be two connected graphs and $\beta\left(G_{1}\right)<\beta\left(G_{2}\right)$. Then,

$$
i_{a v}\left(G_{1}\right)+i_{a v}\left(G_{2}\right)<2 \beta\left(G_{2}\right)
$$

Theorem 5.[2] Let $G_{1}$ and $G_{2}$ be two connected graphs and $i\left(G_{1}\right)<i\left(G_{2}\right)$. Then,

$$
2 i\left(G_{1}\right)<i_{a v}\left(G_{1}\right)+i_{a v}\left(G_{2}\right)
$$

Theorem 6.[1] For two graphs $G_{1}$ and $G_{2}$ of order $m$ and $n$, respectively,

$$
i_{a v}\left(G_{1}+G_{2}\right)=\frac{i_{a v}\left(G_{1}\right) m+i_{a v}\left(G_{2}\right) n}{m+n} .
$$

Theorem 7.[1] For two graphs $G_{1}$ and $G_{2}$ of order $m$ and $n$, respectively,

$$
i_{a v}\left(G_{1}+G_{2}\right) \leq \frac{\beta\left(G_{1}\right) m+\beta\left(G_{2}\right) n}{m+n} .
$$


Theorem 8.[1] For complete graph $K_{n}$ of order $n$ and for any graph $G$ of order $m$,

$$
i_{a v}\left(G \circ K_{n}\right)=m .
$$

\section{Average lower independence number of some splitting graphs}

In this section, we consider the average lower independence number of the splitting graphs $S^{\prime}(G)$ when $G$ is a specified family of graphs. Then, we give the following definition.

Definition 1. For a graph the splitting graph $S^{\prime}(G)$ of graph $G$ is obtained by adding a new vertex $v^{\prime}$ corresponding to each vertex $v$ of $G$ such that $N(v)=N\left(v^{\prime}\right)$ where $N(v)$ and $N\left(v^{\prime}\right)$ are the neighborhood sets of $v$ and $v^{\prime}$, respectively.

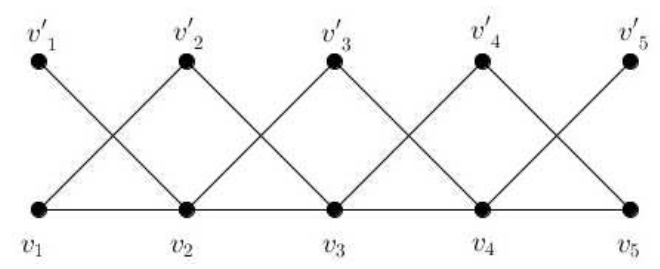

Fig. 1: $S^{\prime}\left(P_{5}\right)$

In order to make the proof of the given theorems understandable, let $S^{\prime}(G)$ be $H$. If we think that the vertex- set of graph $H$ be $V(H)=V_{1}(H) \cup V_{2}(H)$, where

$V_{1}(H)$ : The set contains the vertices of the graph $G$

$V_{2}(H)$ : The set contains the new vertices which are obtained by definition of splitting graph, then it is easily calculated that the average lower independence number of graph $H$.

Theorem 9. Let $P_{n}$ be a path graph with $n$ vertices and $H \cong S^{\prime}\left(P_{n}\right)$ be a splitting path graph with $2 n$ vertices. Then

$$
i_{a v}(H) \geq\left\lceil\frac{2 n}{5}\right\rceil+\frac{1}{2} .
$$

Proof. Every vertex in splitting path graph $H$ included itself adjacent to at most five vertices due to structural of graph $H$. When we calculate the $i_{a v}$ for all $v$ vertices in graph $H$, we should examine the vertices in two cases.

Case 1. Let $v$ be the vertex of the $V_{1}(H)$. We consider the set $i_{v}(H)$ consists of the vertex $v$. The number of vertices of the set $i_{v}(H)$ for $\forall v \in V_{1}(H)$ is greater than or equal to $\left\lceil\frac{2 n}{5}\right\rceil$.

$$
\left|i_{v}(H)\right| \geq\left\lceil\frac{2 n}{5}\right\rceil, \quad \forall v \in V_{1}(H) .
$$

Case 2. Let $v$ be the vertex of the $V_{2}(H)$. We consider the set $i_{v}(H)$ consists of the vertices $v$. The number of vertices of the set $i_{v}(H)$ for $\forall v \in V_{2}(H)$ is greater than or equal to $\left(\left\lceil\frac{2 n}{5}\right\rceil+1\right)$.

$$
\left|i_{v}(H)\right| \geq\left\lceil\frac{2 n}{5}\right\rceil+1, \quad \forall v \in V_{2}(H) .
$$


From Case 1, 2 and the definition of $i_{a v}(H)$, we have

$$
\begin{aligned}
i_{a v}(H) & =\frac{1}{2 n}\left(\sum_{v \in V_{1}(H)} i_{v}(H)+\sum_{v \in V_{2}(H)} i_{v}(H)\right) \\
& \geq \frac{1}{2 n}\left(n\left\lceil\frac{2 n}{5}\right\rceil+n\left(\left\lceil\frac{2 n}{5}\right\rceil+1\right)\right) \\
& \geq\left\lceil\frac{2 n}{5}\right\rceil+\frac{1}{2} .
\end{aligned}
$$

The proof is completed.

Theorem 10. Let $C_{n}$ be a cycle graph of order $n$ and $H \cong S^{\prime}\left(C_{n}\right)$ be a splitting cycle graph of order $2 n$. Then,

$$
\mathbf{1}_{a v}(H)= \begin{cases}\lceil n / 2\rceil+1 / 2 & \text { if } n \equiv_{4} 0 \text { and } n \equiv_{4} 1 \\ \lceil n / 2\rceil+1 & \text { if } n \equiv_{4} 2 \\ \lceil n / 2\rceil & \text { if } n \equiv_{4} 3\end{cases}
$$

Proof. We consider any vertex $v$ of the graph $H$. This vertex is adjacent to at least two vertices and at most four vertices. When we calculate the $i_{a v}$ in graph $H$, we should examine the vertices in three cases.

Case $1\left(n \equiv_{4} 0\right.$ and $\left.n \equiv_{4} 1\right)$. If $n \equiv_{4} 0$ and $n \equiv_{4} 1$, then for $\forall v \in V_{1}(H)$, the $i_{v}(H)$-set including the vertex $v$ has $\left\lceil\frac{n}{2}\right\rceil$ vertices. Similarly, for $\forall v \in V_{2}(H)$, the $i_{v}(H)$-set including the vertex $v$ has $\left\lceil\frac{n}{2}\right\rceil+1$ vertices. From the definition of $i_{a v}(H)$, we have

$$
\begin{aligned}
i_{a v}(H) & =\frac{1}{2 n}\left(\sum_{v \in V_{1}(H)} i_{v}(H)+\sum_{v \in V_{2}(H)} i_{v}(H)\right) \\
& =\frac{1}{2 n}\left(n\left\lceil\frac{n}{2}\right\rceil+n\left(\left\lceil\frac{n}{2}\right\rceil+1\right)\right) \\
& =\left\lceil\frac{n}{2}\right\rceil+\frac{1}{2} .
\end{aligned}
$$

Case $2\left(n \equiv_{4} 2\right)$. For $\forall v \in H$, the number of vertices of the set $i_{v}(H)$ is the same and $\left\lceil\frac{n}{2}\right\rceil+1$. From the definition of $i_{a v}(H)$, we have

$$
\begin{aligned}
i_{a v}(H) & =\frac{1}{2 n}\left(\sum_{v \in V(H)} i_{v}(H)\right) \\
& =\frac{1}{2 n}\left[2 n\left(\left\lceil\frac{n}{2}\right\rceil+1\right)\right] \\
& =\left\lceil\frac{n}{2}\right\rceil+1 .
\end{aligned}
$$

Case $3\left(n \equiv_{4} 3\right)$. For $\forall v \in H$, the number of vertices of the set $i_{v}(H)$ is the same and $\left\lceil\frac{n}{2}\right\rceil$. From the definition of $i_{a v}(H)$, we have

$$
\begin{aligned}
i_{a v}(H) & =\frac{1}{2 n}\left(\sum_{v \in V(H)} i_{v}(H)\right. \\
& =\frac{1}{2 n}\left(2 n\left\lceil\frac{n}{2}\right\rceil\right) \\
& =\left\lceil\frac{n}{2}\right\rceil .
\end{aligned}
$$


The proof is completed.

Theorem 11. Let $K_{n}$ be a complete graph of order $2 n$ and $H \cong S^{\prime}\left(K_{n}\right)$ be a splitting complete graph of order $2 n$. Then, $i_{a v}(H)=2$.

Proof. Let $v$ be the vertex of the $V_{1}(H)$ and new vertex $v^{\prime}$ corresponding to each vertex $v$ in $V_{1}(H)$ be the vertex of the $V_{2}(H)$. We have two cases according to the number of vertices of $H$.

Case 1. The vertex $v$ in $V_{1}(H)$ adjacent to the $n-1$ other vertices of $V_{1}(H)$ and adjacent to every vertex except the vertex $v^{\prime}$ in $V_{2}(H)$. So, the $i_{v}(H)$-set including vertex $v$ doesn't include other vertices of $V_{1}(H)$ and other vertices in $V_{2}(H)$ except the vertex $v^{\prime}$. Thus, we can add the vertex $v^{\prime}$ to the $i_{v}(H)$-set. Hence, the $i_{v}(H)$-set has two vertices namely $v$ and $v^{\prime}$. We have to repeat this process for $n$ vertices of $V_{1}(H)$. Thus, we get $\left|i_{v}(H)\right|=2$.

Case 2. The proof of this case is similar to Case 1. The vertex $v^{\prime}$ in $V_{2}(H)$ adjacent to $n-1$ vertices of $V_{1}(H)$ except corresponding vertex $v$ and none of the vertices in $V_{2}(H)$. To have the $i_{v}(H)$-set, we can add the vertex $v \in V_{1}(H)$ to the $i_{v}(H)$-set. Thus, we can cover every vertex in $V_{2}(H)$ and we have $\left|i_{v}(H)\right|=2$.

Consequently, by Case 1 and Case 2, we have;

$$
\begin{aligned}
\sum_{v \in V(H)} i_{v}(H) & =\sum_{v \in V_{1}(H)} i_{v}(H)+\sum_{v \in V_{2}(H)} i_{v}(H) \\
& =2 n+2 n=4 n .
\end{aligned}
$$

From the definition of $i_{a v}(H)$, we have

$$
\begin{aligned}
i_{a v}(H) & =\frac{1}{|V(H)|} \sum_{v \in V(H)} i_{v}(H) \\
& =\frac{1}{2 n} 4 n=2 .
\end{aligned}
$$

The proof is completed.

Theorem 12. Let $K_{1, n}$ be a star graph of order $n+1$ and let $H \cong S^{\prime}\left(K_{1, n}\right)$ be a splitting star graph of order $2(n+1)$. Then,

$$
i_{a v}(H)=\frac{5 n+4}{2(n+1)} \text {. }
$$

Proof. We should find the value of lower independence number $i_{v}(H)$ of every vertex for average lower independence number of a graph. If we think that the vertex- set of graph $H$ be $V(H)=V_{1}(H) \cup V_{2}(H) \cup V_{3}(H)$, where $V_{1}(H)$ : The set contains $n+1$ vertices of $K_{1, n}$ $V_{2}(H)$ : The set contains vertex $c^{\prime}$ with degree of $n$ which corresponds to the center vertex $c$ in graph $K_{1, n}$. $V_{3}(H)$ : The set contains new vertices except vertex $c^{\prime}$ which added to $K_{1, n}$, then then it is easily calculated that the average lower independence number of graph $H$. We have two cases according to the number of vertices of $H$.

Case 1. Let $v$ be a vertex in $V_{1}(H)$. If $v$ is the center vertex of the $K_{1, n}$, then it is adjacent to $2 n$ vertices in graph $H$. The $i_{v}(H)$-set, which includes vertex $v$ doesn't include other vertices of $V_{1}(H)$ or $V_{3}(H)$. Consequently; to have the $i_{v}(H)$ set; we can add the remaining vertex which corresponds to the vertex $v$. Hence, the $i_{v}(H)$-set has two vertices. Thus, we have $\left|i_{v}(H)\right|=2$.

Let vertex $v$ is any vertex except the center vertex in $V_{1}(H)$. Thus, the $i_{v}(H)$-set, which includes vertex $v$ doesn't include the center vertex $c$ in $V_{1}(H)$ and the vertex $c^{\prime}$ in $V_{2}(H)$. Since the vertex $v$ is adjacent to these vertices, there are 
remaining $V_{1}(H)-\{c\} \cup V_{3}(H)$ vertices in graph $H$. The center vertex $c$ in $K_{1, n}$ is adjacent to $V_{1}(H)-\{c\} \cup V_{3}(H)$ vertices. Thus, we can add the center vertex $c$ to the $i_{v}(H)$-set. Hence, we get $\left|i_{v}(H)\right|=2$ for $\forall v \in V_{1}(H)-\{c\}$.

By writing these values in the definition of $i_{a v}(H)$, we have $i_{v}(H)=2+2 n$.

Case 2. Let $v$ be the only vertex in $V_{2}(H)$. The $i_{v}(H)$-set, which includes vertex $v$, is adjacent to $n$ vertices in $V_{1}(H)-\{c\}$. The remaining vertices in graph $H$ are $V_{3}(H) \cup\{c\}$. Every vertex in $V_{3}(H)$ is adjacent to the center vertex $c$, so we can add only the vertex $c$ to $i_{v}(H)$-set. Hence, we have $\left|i_{v}(H)\right|=2$.

Case 3. Let $v$ be a vertex in $V_{2}(H)$. Thus, the $i_{v}(H)$-set including vertex $v$ is not adjacent any vertex except the center vertex $c$ in $V_{1}(H)$. The remaining vertices in graph $H$ are $V_{1}(H)-\{c\} \cup V_{2}(H) \cup V_{3}(H)$. Due to the structure of the splitting complete graph $H$, the center vertex $c$ is adjacent to every vertex in $V_{1}(H)$ and $V_{3}(H)$. So, the center vertex $c$ is added to $i_{v}(H)=\{v\}$. Then, there is $c^{\prime}$ vertex remaning in $V_{2}(H)$. We can add this vertex to $i_{v}(H)$-set. Hence, we have $\left|i_{v}(H)\right|=|\{v\}|+|\{c\}|+\left|\left\{c^{\prime}\right\}\right|=3$.

We have to repeat this process for $n$ vertices of $V_{3}(H)$. Thus we get $\left|i_{v}(H)\right|=3 n$. Consequently, by Case 1 , Case 2 and Case 3, we have;

$$
\begin{aligned}
i_{a v}(H) & =\frac{1}{|V(H)|} \sum_{v \in V(H)} i_{v}(H) \\
& =\frac{1}{2(n+1)}(3 n+2 n+4)=\frac{5 n+4}{2(n+1)}
\end{aligned}
$$

The proof is completed.

Theorem 13. Let $W_{1, n}$ be a wheel graph of order $n+1$, and let $H \cong S^{\prime}\left(W_{1, n}\right)$ be a splitting wheel graph of order $2(n+1)$. Then, $i_{a v}(H)=\frac{5 n+4}{2(n+1)}$.

Proof. We have to find the value of $i_{v}(H)$ of every vertex in $H$ for average lower independence number. If we think that the vertex set of graph $H$ be $V(H)=V_{1}(H) \cup V_{2}(H) \cup V_{3}(H)$, where

$V_{1}(H)$ : The set contains $n+1$ vertices of $W_{1, n}$,

$V_{2}(H)$ : The set contains vertex $c^{\prime}$ of order $n$ which corresponding vertex of center vertex $c$ in $W_{1, n}$.

$V_{3}(H)$ : The set contains new vertices of order three, except vertex $c^{\prime}$, which added to $W_{1, n}$ by the definition of splitting graph $H$.

Since graph $H$ contains graph $S^{\prime}\left(K_{1, n}\right)$, the proof is done similar to Theorem 12. Hence, average lower independence number of $H$ is $i_{a v}(H)=\frac{5 n+4}{2(n+1)}$. The proof is completed.

\section{Conclusion}

If we want to design a communications network, we wish that it is as possible as stable. Then, we model any communication network by a connected graph. In graph theory, we have many stability measures are called as connectivity, toughness, integrity, domination and its variations. In this paper, we introduce and study the concept of average lower indepence number in graphs, a concept closely related to the problem of finding large independent sets in graphs. In the design of two networks having the same number of processors, if we want to choose the more stable one from these, we take their graph models and it is enough to choose the model whose the average lower independence number is smaller. 


\section{Competing interests}

The authors declare that they have no competing interests.

\section{Authors' contributions}

All authors have contributed to all parts of the article. All authors read and approved the final manuscript.

\section{References}

[1] Aytaç A., Turacı T., The average lower independence number on graph operations, Bulletin of Society of Mathematicians Banja Luka, 16, pp.11-20, 2009.

[2] Aytaç A., Turacı T., Vertex Vulnerability parameter of Gear Graphs, The International Journal of Foundations of Computer Science, 22, no. 5, 1187-1195, 2011.

[3] Aytaç A., Turacı T., The average lower independence number of total graphs , Bull. Int. Math. Virtual Inst. 2, no. 1, 17-27, 2012.

[4] Barefoot, C. A., Entringer, R., Swart, H., Vulnerability in graphs-a comparative survey. J. Combin. Math. Combin. Comput. 1 , 13-22, 1987.

[5] Blidia M., Chellali M. and Maffray F., On Average Lower Independence and Domination Numbers In Graphs, Discrete Math., 295, p. 1-11, 2005.

[6] Bondy J. A. and Murty U. S. R., Graph theory with applications, American Elsevier Publishing Co., Inc., New York, 1976.

[7] Haviland J., Independent domination in regular graphs, Discrte Math.,143, 275-280, 1995.

[8] Henning A. Michael, Trees with equal average domination and independent domination numbers, Ars Combinatoria, 71, pp. 305318, 2004.

[9] Sun L., Wang J. : An upper Bound for the Independent Domination Number, J. of Comb. Theorry series B, 76, 240-246, 1999.

[10] Zhongzhu, Liu, Energy, Laplacian energy and Zagreb index of line graph, middle graph and total graph, Int. J. Contemp. Math. Sci. 5 , no. 17-20, 895?900, 2010. 